\title{
INVARIANCE PROPERTIES OF MILLER-MORITA-MUMFORD CHARACTERISTIC NUMBERS OF FIBRE BUNDLES
}

\author{
THOMAS CHURCH, MARTIN CROSSLEY, AND JEFFREY GIANSIRACUSA
}

\begin{abstract}
Characteristic classes of fibre bundles $E^{d+n} \rightarrow B^{n}$ in the category of closed oriented manifolds give rise to characteristic numbers by integrating the classes over the base. Church, Farb and Thibault [CFT] raised the question of which generalised Miller-Morita-Mumford classes have the property that the associated characteristic number is independent of the fibering and depends only on the cobordism class of the total space $E$. Here we determine a complete answer to this question in both the oriented category and the stably almost complex category. An MMM class has this property if and only if it is a fibre integral of a vector bundle characteristic class that satisfies a certain approximate version of the additivity of the Chern character.
\end{abstract}

\section{INTRODUCTION}

1.1. Background. Let $\pi: E^{n+d} \rightarrow B^{n}$ be a fibre bundle with closed oriented smooth $d$-dimensional manifold fibres. The generalised Miller-Morita-Mumford (MMM) characteristic classes for such bundles are a family of characteristic classes living in the cohomology of the base. They were first introduced in [Mil86], [Mor87], and [Mum83], and there is an extensive literature on these classes when $d=2$, including the proof of Mumford's Conjecture and work on Faber's Conjectures. In higher dimensions these classes are somewhat less studied, though there are some interesting results, such as those of [Ebe11b], [Ebe11a].

In [CFT] the first author proved with Farb and Thibault that, in the $d=2$ setting, certain characteristic numbers associated with the MMM classes could be expressed purely in terms of the oriented bordism class of the total space $E$. The purpose of this paper is to explore this phenomenon more thoroughly from the point of view of stable homotopy and bordism theory.

The generalised MMM classes are defined as follows. A characteristic class $X \in H^{*}(B S O(d) ; \mathbb{Z})$ for rank $d$ vector bundles (rationally $X$ can be written as a polynomial in the Euler class and Pontrjagin classes $\left.p_{1}, \ldots, p_{\lfloor d / 2\rfloor}\right)$ can be evaluated on the fibrewise tangent bundle $T^{v} E \rightarrow E$ to produce a cohomology class $X\left(T^{v} E\right) \in H^{*}(E ; \mathbb{Z})$. The image of this class under the pushforward map $\pi_{!}: H^{*}(E ; \mathbb{Z}) \rightarrow H^{*-d}(B ; \mathbb{Z})$ is a class

$$
\widehat{X}(E \stackrel{\pi}{\rightarrow} B):=\pi_{!} X\left(T^{v} E\right) \in H^{*}(B ; \mathbb{Z})
$$

(note that $\widehat{X}$ is zero if $\operatorname{deg}(X)<d$ ) that is natural with respect to pullbacks of bundles. Products and sums of these classes are also natural with respect to pullbacks. The polynomials in the classes $\widehat{X}$ produced by this construction are, by definition, the generalised MMM classes for $d$-dimensional oriented fibre bundles. These classes form an algebra $\mathscr{C}_{d}$ (over $\mathbb{Z}$ ). More precisely, $\mathscr{C}_{d}$ is the free graded commutative algebra generated by $H^{>d}(B S O(d) ; \mathbb{Z}$ ) (with degrees shifted down by $d$ so that a degree $d+i$ class gives a generator of degree $i$ ). We promote it to a bialgebra by declaring the generators to be primitive.

Date: 15 December, 2011. 
If the base $B$ is a closed oriented manifold of dimension $n$ and $x \in \mathscr{C}_{d}$ is of degree $n$ then we define an associated characteristic number $x^{\sharp}$ by pairing with the fundamental class of the base,

$$
x^{\sharp}(E \rightarrow B):=\langle x(E \rightarrow B),[B]\rangle \in \mathbb{Z} .
$$

The characteristic class $x(E \rightarrow B)$ is an invariant living in a group that depends on the base $B$, and so the characteristic classes of bundles over different bases cannot be directly compared. However, the associated characteristic numbers, being simply integers, do allow for immediate comparison.

At this point it is useful to observe that the characteristic number $x^{\sharp}(E \rightarrow B)$ depends only on the class of $x$ in $\mathscr{C}_{d} \otimes \mathbb{Q}$; the 2-torsion classes in $H^{*}(B S O(d) ; \mathbb{Z})$ (there is no other torsion) yield MMM classes that necessarily give zero when integrated over the base. Thus, when studying MMM characteristic numbers, one loses no information by working rationally, and we shall do this from here onwards.

It sometimes happens that a given manifold $E^{d+n}$ admits multiple distinct fiberings $E \rightarrow B$ over bases of a fixed dimension. In [CFT] the following question is raised. To what extent do the MMM characteristic numbers actually depend on the fibering? The main result of that paper is that, in the case of surface bundles, if $x$ is the MMM class $e_{2 n-1}:=\pi_{!}\left(e\left(T^{v} E\right)^{2 n}\right) \in H^{2 n-2}(B)$ determined by an even power of the Euler class then $x^{\sharp}$ can be written as a polynomial in the Pontrjagin numbers of $E$ and thus depends only on the oriented topological cobordism class of $E$ and is completely independent of the fibering.

In light of the question and results of [CFT], it then is natural to try to decide which of the generalised MMM classes have associated characteristic numbers that can be expressed purely as functions of the bordism class of the total space. To state this problem more precisely we introduce some notation. Let $B_{u n}$ denote the set of isomorphism classes of bundles with $d$-dimensional fibres in the category of smooth closed oriented manifolds; this is a commutative monoid with respect to disjoint union. Sending a bundle to the bordism class of its total space defines an additive map Tot: Bun $_{d} \rightarrow M S O_{*}(p t)$. A class $x \in \mathscr{C}_{d} \otimes \mathbb{Q}$ determines an additive map $x^{\sharp}: B_{u} n_{d} \rightarrow \mathbb{Q}$. In this paper we give a complete answer to the following question.

Question 1.1.1. For which classes $x \in \mathscr{C}_{d} \otimes \mathbb{Q}$ does there exist a factorisation as below?

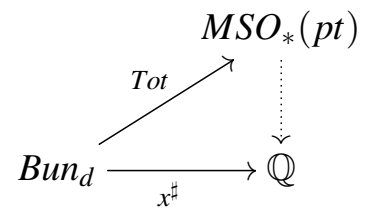

1.2. The motivating example. Let us first observe that there is an obvious family of elements in $\mathscr{C}_{d}$ for which the associated characteristic numbers depend only on the total space of the bundle. Recall that $H^{*}(B S O ; \mathbb{Q})$ is a Hopf algebra with coproduct $\Delta$ induced by the direct sum map. Suppose $X \in H^{*}(B S O ; \mathbb{Q})$ is primitive (meaning that $\left.\Delta(X)=X \otimes 1+1 \otimes X\right)$ and consider the corresponding generalised MMM class $\widehat{X} \in \mathscr{C}_{d} \otimes \mathbb{Q}$ and its associated characteristic number $\widehat{X}^{\sharp}$. Given a bundle $\pi: E^{n+d} \rightarrow B^{n}$, there is a short exact sequence $T^{v} E \rightarrow T E \rightarrow \pi^{*} T B$ and hence $X(T E)=X\left(T^{v} E\right)+\pi^{*} X(T B)$ since $X$ is primitive. We then have,

$$
\begin{aligned}
\langle X(T E),[E]\rangle & =\left\langle\pi_{!} X(T E),[B]\right\rangle \\
& =\left\langle\pi_{!} X\left(T^{v} E\right),[B]\right\rangle+\left\langle\pi_{!} \pi^{*} X(T B),[B]\right\rangle \\
& =\widehat{X}^{\sharp}(E \rightarrow B)
\end{aligned}
$$

since $\pi_{!} \circ \pi^{*}$ is the zero map. Thus $\widehat{X}^{\sharp}(E \rightarrow B)$ can be expressed as a polynomial in the tangential Pontrjagin numbers of $E$ which depend only on the class of $E$ in oriented bordism tensored with $\mathbb{Q}$. 
The primitives in $H^{*}(B S O ; \mathbb{Q})$ are easily described. The Pontrjagin character $P h \in H^{*}(B S O ; \mathbb{Q})$ is the pullback of the Chern character $C h$ via the complexification map $B S O \rightarrow B U$. The Chern character is additive: $C h(V \oplus W)=C h(V)+C h(W)$, and so the Pontrjagin character is also additive; i.e., the components are primitive. The space of primitives in $H^{*}(B U ; \mathbb{Q})$ and $H^{*}(B S O ; \mathbb{Q})$ is spanned by the components of the Chern character and Pontrjagin character, respectively, and the primitives in fact freely generate the cohomology as a ring since the cohomology is a commutative and cocommutative connected Hopf algebra.

When $d=2$,

$$
\mathscr{C}_{2} \cong \mathbb{Z}\left[e_{1}, e_{2}, \ldots\right],
$$

where $e_{i}=\widehat{e^{i+1}}$. The restriction of the Pontrjagin character to $B S O(2)$ is zero in degrees $4 i+2$ and is proportional to $e^{2 i}=p_{1}^{i}$ in degree $4 i$. Thus the $e_{\text {odd }}$ characteristic numbers are invariants of the total space, recovering the main theorem of $[\overline{C F T}]$. It turns out, as we shall see in Theorem $D$ below, that these are the only characteristic numbers having this property.

1.3. The method. The main idea of this paper is to rephrase Question 1.1.1 in terms of stable homotopy and certain bordism spectra. We first show that the maps $x^{\sharp}: B u n_{d} \rightarrow \mathbb{Q}$ and Tot: $\operatorname{Bun}_{d} \rightarrow M S O_{*}(p t)$ both admit canonical factorisations through an additive map

$$
\alpha: \mathrm{Bun}_{d} \rightarrow \mathrm{MSO}_{*}\left(\Omega^{\infty} \mathbf{M T S O}(d)\right),
$$

where $\operatorname{MTSO}(d)$ is the Madsen-Tillmann spectrum (defined in Section2 below); we describe all of these maps at the level of spectra. In the diagram,

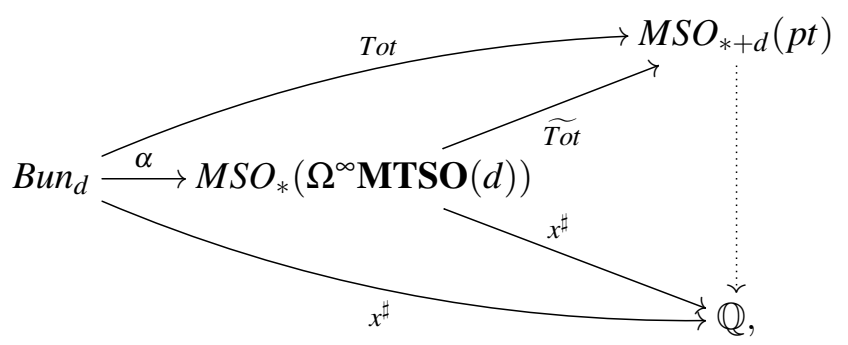

a straightforward rational calculation in stable homotopy then determines exactly when the dotted arrow can be completed to make the inner triangle commute. Given this, we show that the results of Ebert [Ebe11a] determine enough information about the image of $\alpha$ to decide exactly when the dotted arrow can be filled to make the outer triangle commute.

1.4. Near-primitive characteristic classes of vector bundles. In carrying out the rational calculation in Section 5 we find that the argument given above in Section 1.2 with the Pontrjagin character (when reformulated in terms of stable homotopy) actually yields the factorisation asked for in Question 1.1 .1 for a class of elements in $H^{*}(B S O ; \mathbb{Q})$ that is somewhat larger than the space of primitives; we call these new elements near-primitives.

Definition 1.4.1. Let $H$ be a graded connected cocommutative Hopf algebra. A near-primitive of order $d$ in $H$ is an element $x$ of degree at least $d$ such that the composition

$$
H \stackrel{\Delta}{\rightarrow} H \otimes H \stackrel{i d \otimes \text { proj }}{\longrightarrow} H \otimes H / H^{<d}
$$

sends $x$ to $1 \otimes x$.

Note that if $H$ is concentrated in nonnegative degrees then a near-primitive of order 1 is simply a primitive. In Section 6 we give a complete classification of near primitives in $H^{*}(B S O ; \mathbb{Q})$ and $H^{*}(B U ; \mathbb{Q})$. 
Theorem A. The space of order d near-primitives in $H^{*}(B S O ; \mathbb{Q})$ or $H^{*}(B U ; \mathbb{Q})$ has a basis consisting of those monomials in the primitives such that all proper factors have degree strictly less than d. Explicitly, the degree $m$ component of the space is equal to the degree $m$ part of $\mathbb{Q}\left[Q_{i}|m-d<| Q_{i} \mid<d\right] \oplus \mathbb{Q} Q_{m}$, where the $Q_{i}$ are the components of the Pontrjagin character or Chern character respectively.

A primitive is a near-primitive (of any order); conversely, it follows from the theorem that the only near-primitives of order $d$ in degrees $\geq 2 d$ are the ordinary primitives. In particular, for $B S O$, when $d=2$ all near-primitives are primitive and their restrictions to $B S O(2)$ are proportional to the odd powers of the Euler class. However, in the range $d \leq *<2 d$ the space of near-primitives can be significantly larger than the subspace of primitives. The simplest example of near-primitives that are not primitive occurs when $5 \leq d \leq 8$; in this case all elements of degree 8 (all elements in the span of $p_{1}^{2}$ and $p_{2}$ ) are near-primitive of order $d$, while only the scalar multiples of $p_{2}-(1 / 2) p_{1}^{2}$ are primitive.

1.5. Results. The result of our rational calculation is the following determination of when the inner triangle in diagram (1.3.1) can be completed.

Theorem B. Given a class $x \in \mathscr{C}_{d}$, the map $x^{\sharp}: \operatorname{MSO}_{*}\left(\Omega^{\infty} \operatorname{MTSO}(d)\right) \rightarrow \mathbb{Q}$ factors through $\widetilde{T o t}: \operatorname{MSO}_{*}\left(\Omega^{\infty} \mathbf{M T S O}(d)\right) \rightarrow M S O_{*}(p t)$ if and only if $x$ satisfies the following conditions:

(1) $x$ is primitive, so it is of the form $\widehat{X}$ for some $X \in H^{*}(B S O(d) ; \mathbb{Q})$, and

(2) $X$ is the restriction of a near-primitive of order $d$ in $H^{*}(B S O ; \mathbb{Q})$.

We now turn to the outer triangle in diagram (1.3.1). Let $L \subset H^{*}(B S O(d) ; \mathbb{Q})$ denote the subspace spanned by the components of the Hirzebruch $L$-class (restricted to $B S O(d)$ ) and let $K$ denote the ideal in $\mathscr{C}_{d} \otimes \mathbb{Q}$ generated by the elements of the form $\widehat{X}$ for $X \in L$. Let $N P_{d} \subset H^{*}(B S O(d) ; \mathbb{Q})$ denote the image of the space of near-primitives of order $d$ on $B S O$ under restriction from $B S O$ to $B S O(d)$. Our main result is the following:

Theorem C. Given a class $x \in \mathscr{C}_{d} \otimes \mathbb{Q}$, the map $x^{\sharp}:$ Bun $_{d} \rightarrow \mathbb{Q}$ factors through Tot: Bun ${ }_{d} \rightarrow$ $M S O_{*}(p t)$ if and only if there exists $X \in N P_{d}$ such that $x=\widehat{X}$ in $\mathscr{C}_{d}$ when $d$ is even and in $\mathscr{C}_{d} / K$ when $d$ is odd.

As a special case of this theorem, when $d=2$ we find that the sufficient condition given in [CFT] is in fact also necessary.

Corollary D. Given $x \in \mathscr{C}_{2} \otimes \mathbb{Q} \cong \mathbb{Q}\left[e_{1}, e_{2}, \ldots\right]$, the characteristic number $x^{\sharp}:$ Bun $n_{2} \rightarrow \mathbb{Q}$ factors through Tot: $\mathrm{Bun}_{2} \rightarrow \mathrm{MSO}_{*}(\mathrm{pt})$ if and only if $x$ is in the span of the odd MMM class $\left\{e_{2 i+1}\right\}$.

1.6. Almost complex bundles. There is an analogous story in the setting of almost complex fibre bundles. Consider the set $B u n_{d}^{\mathbb{C}}$ of smooth fibre bundles $\pi: E \rightarrow B$ in which

(1) the fibrewise tangent bundle $T^{v} E$ is equipped with a complex structure,

(2) both $B$ and $E$ are closed oriented stably almost complex manifolds (i.e., they have almost complex structures on their stable tangent bundles),

(3) and the short exact sequence $T^{v} E \rightarrow T E \rightarrow \pi^{*} T B$ of real bundles becomes a short exact sequence of complex vector bundles after an appropriate stabilisation. 
Replacing $S O(d)$ with $U(d)$, we let $\mathscr{C}_{d}^{\mathbb{C}}$ denote the algebra of generalised MMM classes in this context. For any $x \in \mathscr{C}_{d}^{\mathbb{C}}$ we consider the diagram

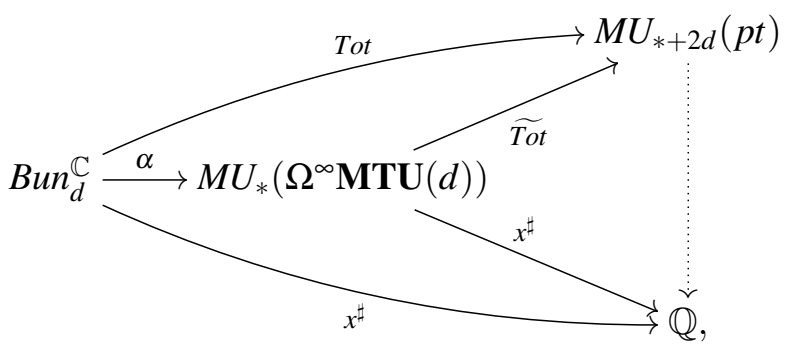

and we find a corresponding theorem.

Theorem E. For any $x \in \mathscr{C}_{d}^{\mathbb{C}}$ the following statements hold.

(1) The map $x^{\sharp}: \operatorname{MU}_{*}\left(\Omega^{\infty} \mathbf{M T U}(d)\right) \rightarrow \mathbb{Q}$ factors through the map $\widetilde{\text { Tot }}: M U_{*}\left(\Omega^{\infty} \mathbf{M T U}(d)\right) \rightarrow$ $M U_{*}(p t)$ if and only if $x$ is of the form $\widehat{X}$ for some $X \in H^{*}(B U(d) ; \mathbb{Q})$ that is the restriction of a near-primitive of order $2 d$ in $H^{*}(B U ; \mathbb{Q})$.

(2) The same is true for factoring the map $x^{\sharp}:$ Bun $_{d}^{\mathbb{C}} \rightarrow \mathbb{Q}$ through Tot: Bun $_{d} \rightarrow M_{*}(p t)$.

Consider the special case of the above theorem for holomorphic surface bundles, i.e., when $d=1$. As in the real oriented case, $\alpha^{\mathbb{C}}: B u n_{d}^{\mathbb{C}} \rightarrow M U_{*}\left(\Omega^{\infty} \mathbf{M T U}(1)\right)$ is surjective and $\mathscr{C}_{1}^{\mathbb{C}}=\mathbb{Z}\left[e_{1}, e_{2}, \ldots\right]$, but now $e^{i}$ is proportional to a component of the Chern character no matter whether $i$ is even or odd - in fact, $\left.C h\right|_{B U(1)}=\sum_{n}(1 / n !) e^{n}$. Thus all of the primitive classes in $\mathscr{C}_{1}^{\mathbb{C}}$ give characteristic numbers for holomorphic surface bundles that depend only on the complex cobordism class of the total space, and these are the only classes with this property.

\section{THOM SPECTRA, MADSEN-TILLMANN SPECTRA AND BORDISM}

We begin by recalling some well-known properties of Thom spectra and their relation to bordism theory.

2.1. Thom spectra. Recall that the Thom space $\operatorname{Th}(\xi)$ of a vector bundle $\xi \rightarrow B$ is the disc bundle modulo its boundary sphere bundle, $D(\xi) / S(\xi)$. Since the Thom space of a rank $n$ trivial bundle is homeomorphic to the $n$-fold suspension of the base, we define the Thom $\operatorname{spectrum~} \mathbf{T h}\left(\xi-\mathbb{R}^{n}\right)$ of a virtual bundle of the form $\xi-\mathbb{R}^{n}$ to be $\Sigma^{-n} \mathrm{Th}(\xi)$; this construction extends to arbitrary $K O$-theory classes over CW bases via the skeletal filtration of the base. The Thom space and Thom spectrum send external products to smash products, and they are functorial with respect to bundle pullbacks: given a map $f: X \rightarrow Y$ and a class $\zeta \in K O(Y)$, there is a map $\mathbf{T h}\left(f^{*} \zeta\right) \rightarrow \mathbf{T h}(\zeta)$. Since a sum $\zeta \oplus \eta$ is the pullback of the external product $\zeta \times \eta$ on $X \times X$ by the diagonal, there is a map $\operatorname{Th}(\zeta \oplus \eta) \rightarrow \mathbf{T h}(\zeta) \wedge \mathbf{T h}(\eta)$. In particular, when $\eta=0$ in $K O(X)$ we get a map

$$
\Delta: \mathbf{T h}(\zeta) \rightarrow \mathbf{T h}(\zeta) \wedge X_{+}
$$

(which makes $\mathbf{T h}(\zeta)$ into a comodule spectrum over the coalgebra $\Sigma^{\infty} X_{+}$with its diagonal map).

Let $\gamma_{n, m}$ denote the tautological $n$-plane bundle over the Grassmannian $G r_{n}^{+}\left(\mathbb{R}^{n+m}\right)$ of real oriented $n$-planes in $\mathbb{R}^{n+m}$ (when $m=\infty$ we will write $\gamma_{n} \rightarrow B S O(n)$ ). One defines the MadsenTillmann spectrum

$$
\operatorname{MTSO}(n):=\operatorname{Th}\left(-\gamma_{n}\right)
$$


Explicitly, the $(n+k)^{t h}$ space of this spectrum is $\operatorname{Th}\left(\gamma_{n, k}^{\perp}\right)$, and the spectrum structure maps are induced from the identification $\left.\gamma_{n, k+1}\right|_{G r_{n}^{+}\left(\mathbb{R}^{n+k}\right)} \cong \gamma_{n, k} \oplus \mathbb{R}$. By the functoriality of Thom spectra, the classifying map $B \rightarrow B S O(d)$ for a rank $d$ vector bundle $\xi$ determines a map of spectra

$$
C_{\xi}: \operatorname{Th}(-\xi) \rightarrow \operatorname{MTSO}(d) .
$$

Since $\left.\gamma_{k+1}\right|_{G r_{k}^{+}\left(\mathbb{R}^{\infty}\right)} \cong \gamma_{k} \oplus \mathbb{R}$, the $K O$-theory classes $\left\{\gamma_{n}-\mathbb{R}^{n}\right\}_{n \in \mathbb{N}}$ assemble to a class $\gamma$ on $B S O=\operatorname{colim}_{n} B S O(n)$ of virtual dimension zero. One defines Thom's famous spectrum MSO as

$$
\text { MSO }:=\mathbf{T h}(\gamma)
$$

Concretely, it is the spectrum with $k^{\text {th }}$ space $\operatorname{Th}\left(\gamma_{k}\right)$ and structure maps

$$
\Sigma \operatorname{Th}\left(\gamma_{k}\right) \cong \operatorname{Th}\left(\left.\gamma_{k+1}\right|_{G r_{k}^{+}\left(\mathbb{R}^{\infty}\right)}\right) \hookrightarrow \operatorname{Th}\left(\gamma_{k+1}\right) .
$$

By the functoriality of Thom spectra, the classifying map $X \rightarrow B S O$ for a class $\xi \in K O(X)$ of virtual dimension $n$ produces a map of Thom spectra

$$
C_{\xi}: \mathbf{T h}(\xi) \rightarrow \Sigma^{n} \text { MSO }
$$

We call attention to two special cases of this map. First, the virtual bundle $-\gamma_{n}$ over $G r_{n}^{+}\left(\mathbb{R}^{\infty}\right)$ gives a map of spectra, $C_{-\gamma_{n}}: \operatorname{MTSO}(n) \rightarrow \Sigma^{-n}$ MSO. Second, the external product $\gamma \times \gamma$ over $B S O \times B S O$ gives a product map $\mu=C_{\gamma \times \gamma}: \mathbf{M S O} \wedge$ MSO $\rightarrow$ MSO making MSO into a ring spectrum.

2.2. Pre-transfers. Given a bundle $\pi: E \rightarrow B$, there is a pre-transfer map

$$
P T_{\pi}: \Sigma^{\infty} B_{+} \rightarrow \mathbf{T h}\left(-T^{v} E\right) .
$$

It is constructed by choosing a fibrewise embedding of $E$ into a trivial vector bundle over $B$ with a tubular neighbourhood, collapsing everything outside the tubular neighbourhood to the basepoint, and then identifying the tubular neighbourhood with the open disc bundle of the fibrewise normal bundle. The pre-transfer is well defined up to homotopy.

For later use we record the following factorisation property of pre-transfers.

Lemma 2.2.1. Given a smooth bundle of closed oriented d-manifolds $\pi: E \rightarrow B$, the diagram

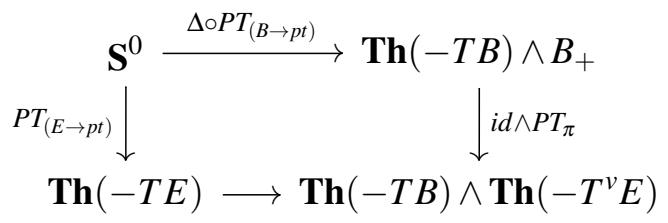

(with bottom arrow determined by the splitting $\left.T E \cong \pi^{*} T B \oplus T^{v} E\right)$ ) commutes up to homotopy.

Proof. Let $V, W$ be real vector spaces of sufficiently large dimension so that we can choose an embedding of $\phi: B \hookrightarrow V$ and a fibrewise embedding $\psi: E \hookrightarrow B \times W$ over $B$. Let $\Psi=(\phi \times$ id) $\circ \psi: E \hookrightarrow V \times W$. Let $N \Psi, N \psi, N \phi$ be the respective normal bundles of $\Psi, \psi$ and $\phi$. There is a canonical splitting $N \Psi \cong \pi^{*} N \phi \oplus N \psi$. By choosing appropriate tubular neighbourhoods and identifications with the disc bundles one obtains a diagram

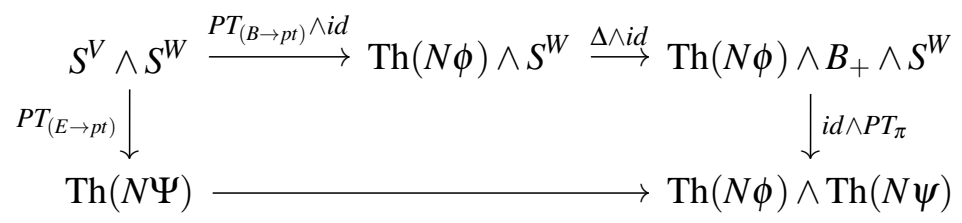


that commutes on the nose. (The PT maps here are unstable representatives of the respective pre-transfers constructed from the chosen embeddings and tubular neighbourhoods.) The diagram in the statement of the lemma is the stabilisation of this.

2.3. Oriented Bordism. The classical Pontrjagin-Thom construction identifies the generalised homology theory $\mathrm{MSO}_{*}(X)=\pi_{*}\left(\mathbf{M S O} \wedge X_{+}\right)$associated with the spectrum MSO as oriented bordism $-\mathrm{MSO}_{n}(X)$ is the abelian group of oriented bordism classes of $n$-manifolds mapping to $X$, with the group structure given by disjoint union. Let us recall how the half of the Pontrjagin-Thom construction that goes from geometric cycles to homotopy classes can be stated efficiently in terms of pre-transfers.

The oriented bordism class of a manifold $M^{n}$, as an element of $\operatorname{MSO}_{n}(p t)=\pi_{n} \mathbf{M S O}$, is represented by the composition

$$
\mathbf{S}^{0}=\Sigma^{\infty} p t_{+} \stackrel{P T_{(M \rightarrow p t)}}{\longrightarrow} \mathbf{T h}(-T M) \stackrel{C_{-T M}}{\longrightarrow} \Sigma^{-n} \text { MSO. }
$$

More generally, if $M$ is equipped with a map $f$ to a target space $X$, then the class $[f: M \rightarrow X] \in$ $\mathrm{MSO}_{n}(X)$ is represented by the composition

$$
\mathbf{S}^{0} \rightarrow \Sigma^{\infty} p t_{+} \stackrel{P T_{(M \rightarrow p t)}}{\longrightarrow} \mathbf{T h}(-T M) \stackrel{\Delta}{\longrightarrow} \mathbf{T h}(-T M) \wedge M_{+} \stackrel{C_{-T M} \wedge f}{\longrightarrow} \Sigma^{-n} \mathbf{M S O} \wedge X_{+} .
$$

2.4. Classifying maps for fibre bundles and the map $\alpha$. Via (parametrised) Pontrjagin-Thom theory, the infinite loop space $\Omega^{\infty} \mathbf{M T S O}(d)$ classifies bundles of smooth oriented closed $d$ manifolds up to an an appropriate equivalence. We shall make use of these classifying maps to define the map $\alpha:$ Bun $_{d} \rightarrow \mathrm{MSO}_{*}\left(\Omega^{\infty} \mathbf{M T S O}(d)\right)$ discussed in the introduction. The key point is that this map sends a bundle to a class that determines both the oriented bordism class of the total space of the bundle and all MMM characteristic numbers of the bundle.

A closed oriented $d$-manifold $M$ determines an element $[M] \in \pi_{0} \mathbf{M T S O}(d)=\pi_{0} \Omega^{\infty} \mathbf{M T S O}(d)$ by the obvious modification of the construction from the preceding section. More generally, a bundle of $d$-manifolds $\pi: E^{d+n} \rightarrow B^{n}$ determines a homotopy class

$$
\alpha_{\pi}: B \rightarrow \Omega^{\infty} \mathbf{M T S O}(d) .
$$

given by the adjoint of $C_{-T^{v} E} \circ P T_{\pi}$. From this description one sees immediately that $\alpha_{\pi}$ is natural (up to homotopy) with respect to pullbacks.

Let us comment on connected components. Since $\Omega^{\infty} \mathbf{M T S O}(d)$ is a group-like $H$-space, each component can be canonically identified (up to homotopy) with the identity component $\Omega_{0}^{\infty} \mathbf{M T S O}(d)$. By [Ebe11b, Theorem A.0.3], the group $\pi_{0} \Omega^{\infty} \mathbf{M T S O}(d)$ fits into a split short exact sequence

$$
0 \rightarrow \mathbb{Z} / k_{d+1} \mathbb{Z} \rightarrow \pi_{0} \Omega^{\infty} \text { MTSO }(d) \rightarrow \pi_{d} \text { MSO }
$$

where $k_{d}$ is 0 if $d$ is odd, 1 if $d \equiv 0(\bmod 4)$, and 2 if $d \equiv 2(\bmod 4)$. On each component of $B$ the map $\alpha_{\pi}$ lands in the component of $\Omega^{\infty} \operatorname{MTSO}(d)$ corresponding to the class of the fibre over that component.

If the base $B$ is a closed oriented $n$-manifold, we can regard the above map $\alpha_{\pi}$ as representing a class

$$
\left[\alpha_{\pi}\right] \in \operatorname{MSO}_{n}\left(\Omega^{\infty} \mathbf{M T S O}(d)\right)
$$

in the group of oriented bordism classes of $n$-manifolds mapping to $\Omega^{\infty} \operatorname{MTSO}(d)$. The map $\alpha: \mathrm{Bun}_{d} \rightarrow \mathrm{MSO}_{*}\left(\Omega^{\infty} \mathbf{M T S O}(d)\right)$ is defined by the rule $(E \stackrel{\pi}{\rightarrow} B) \mapsto\left[\alpha_{\pi}\right]$. 
2.5. The oriented bordism class of the total space. The oriented cobordism class of the total space $E$ is an element $[E] \in M S O_{n+d}(p t)$. Here we show how $[E]$ is determined by $\left[\alpha_{\pi}\right]$ and hence give a canonical factorisation of Tot $: \operatorname{Bun}_{d} \rightarrow M_{*} O_{*+d}(p t)$ as $\operatorname{Bun}_{d} \stackrel{\alpha}{\rightarrow} M S O_{*}\left(\Omega^{\infty} \operatorname{MTSO}(d)\right) \stackrel{\widetilde{\text { Tot }} \rightarrow}{\rightarrow}$ $M S O_{*+d}(p t)$.

The counit of the $\Omega^{\infty}-\Sigma^{\infty}$ adjunction gives a map of spectra $\sigma: \Sigma^{\infty} \Omega^{\infty} \mathbf{M T S O}(d)_{+} \rightarrow \mathbf{M T S O}(d)$. Using the map MTSO $(d) \rightarrow \Sigma^{-d}$ MSO and the ring spectrum product of MSO, the composition

$$
\operatorname{MSO} \wedge \Omega^{\infty} \operatorname{MTSO}(d)_{+} \rightarrow \operatorname{MSO} \wedge \operatorname{MTSO}(d) \rightarrow \operatorname{MSO} \wedge \Sigma^{-d} \text { MSO } \rightarrow \Sigma^{-d} \text { MSO }
$$

induces an additive map

$$
\widetilde{\text { Tot }}: \operatorname{MSO}_{n}\left(\Omega^{\infty} \mathbf{M T S O}(d)\right) \rightarrow M O_{n+d}(p t) .
$$

Lemma 2.5.1. The equality $\widetilde{\operatorname{Tot}}\left(\left[\alpha_{\pi}\right]\right)=[E]$ holds.

Proof. The oriented bordism class $[E] \in \pi_{n+d} \mathbf{M S O}$ is represented by the map $C_{-T E} \circ P T_{(E \rightarrow p t)}: \mathbf{S}^{0} \rightarrow$ $\Sigma^{-n-d}$ MSO. Since $T E \cong T^{v} E \oplus \pi^{*} T B$, this map factors as

$$
\mathbf{S}^{0} \stackrel{P T_{(E \rightarrow p t)}}{\longrightarrow} \mathbf{T h}\left(-\pi^{*} T B-T^{v} E\right) \longrightarrow \mathbf{T h}(-T B) \wedge \mathbf{T h}\left(-T^{v} E\right) \stackrel{\mu \circ C_{(-T B) \times\left(-T^{v} E\right)}}{\longrightarrow} \Sigma^{-n-d} \text { MSO. }
$$

Since $\alpha_{\pi}$ is the adjoint of $C_{-T^{v} E} \circ P T_{\pi}$ and $\sigma$ is the adjoint of the identity map on $\Omega^{\infty} \operatorname{MTSO}(d)$, it follows that the diagram

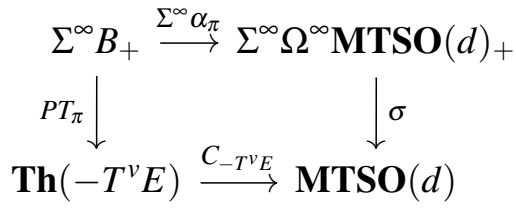

commutes up to homotopy. The claim now follows directly from the definitions, the factorisation 2.5.1, the diagram 2.5.2 and Lemma 2.2.1.

\section{Generalised MMM Classes Via MAdSEn-Tillmann SPECTRA}

3.1. Generalised MMM classes. Here we recall how one can think of the generalised MMM classes of $d$-dimensional fibre bundles universally as classes in the cohomology of $\Omega^{\infty} \mathbf{M T S O}(d)$.

Proposition 3.1.1. There is a canonical injective morphism $\mathscr{C}_{d} \hookrightarrow H^{*}\left(\Omega_{0}^{\infty} \mathbf{M T S O}(d) ; \mathbb{Z}\right)$ of bialgebras, and after tensoring with $\mathbb{Q}$ it becomes an isomorphism.

Proof. By the Thom isomorphism, the (integral) spectrum cohomology of $\operatorname{MTSO}(d)$ is a free $H^{*}(B S O(d) ; \mathbb{Z})$-module on one generator $u$ (the Thom class) in degree $-d$. The map $\sigma$ from the previous section induces a map on cohomology,

$$
\sigma^{*}: H^{*}(\operatorname{MTSO}(d) ; \mathbb{Z}) \rightarrow H^{*}\left(\Omega^{\infty} \mathbf{M T S O}(d) ; \mathbb{Z}\right) .
$$

Let $\sigma_{0}^{*}$ denote the composition of $\sigma^{*}$ followed by restriction to the identity component $\Omega_{0}^{\infty} \mathbf{M T S O}(d)$; $\sigma_{0}^{*}$ is injective in positive degrees and it is trivially zero in negative degrees. The morphism of the proposition is defined by sending the MMM class $\widehat{X}=\pi_{!} X$ to $\sigma_{0}^{*}(u X)$. With rational coefficients the positive degree part of the image of $\sigma_{0}^{*}$ coincides with the space of primitives and this subspace freely generates the cohomology $H^{*}\left(\Omega_{0}^{\infty} \mathbf{M T S O}(d) ; \mathbb{Q}\right)$ as a graded commutative algebra by the Milnor-Moore theorem [MM65, p. 263]. 
Note that in positive degrees the image of $\sigma^{*}$ is contained in the subspace of $\pi_{0} \Omega^{\infty} \mathbf{M T S O}(d)$ invariants. The cohomology of the component $\Omega_{0}^{\infty} \mathbf{M T S O}(d)$ is canonically isomorphic to the $\pi_{0}$-invariant subspace of the cohomology of $\Omega^{\infty} \mathbf{M T S O}(d)$. Hence we shall identify $\mathscr{C}_{d} \otimes \mathbb{Q}$ with the $\pi_{0}$-invariants in $H^{*}\left(\Omega^{\infty} \mathbf{M T S O}(d) ; \mathbb{Q}\right)$.

Given a bundle $\pi: E \rightarrow B$ of closed oriented manifolds and a vector bundle characteristic class $X \in H^{*}(B S O(d) ; \mathbb{Z})$, we have a cohomology class $u X \in H^{*}(B S O(d) ; \mathbb{Z})[u] \cong H^{*}(\operatorname{MTSO}(d) ; \mathbb{Z})$ and also a primitive generalised MMM class $\pi_{!} X\left(T^{v} E\right)$. This proposition justifies our identifications above.

Proposition 3.1.2. For any bundle $\pi: E \rightarrow B$ there is an equality, $\left(\alpha_{\pi}\right)^{*} \sigma^{*}(u X)=\pi_{!} X\left(T^{v} E\right)$.

Proof. This follows immediately from the commutativity of the diagram 2.5.2) and the fact that the pre-transfer in cohomology, $P T_{\pi}^{*}$, composed with the Thom isomorphism for $-T^{v} E$ is equal to the pushforward map $\pi_{!}$.

3.2. MMM characteristic numbers from $\left[\alpha_{\pi}\right]$. We now show how the generalised MMM characteristic numbers for a bundle $\pi: E^{n+d} \rightarrow B^{n}$ are determined by the class $\left[\alpha_{\pi}\right] \in M S O_{n}\left(\Omega^{\infty} \mathbf{M T S O}(d)\right)$ constructed earlier. Hence a generalised MMM characteristic number $x^{\sharp}$ admits a canonical factorisation $\operatorname{Bun}_{d} \stackrel{\alpha}{\rightarrow} \mathrm{MSO}_{*}\left(\Omega^{\infty} \mathbf{M T S O}(d)\right) \rightarrow \mathbb{Q}$.

Let $\mathbf{H} \mathbb{Z}$ denote the Eilenberg-MacLane spectrum representing ordinary cohomology. The Thom class of $M S O$ determines a map of ring spectra

$$
\text { th }: \mathbf{M S O} \rightarrow \mathbf{H} \mathbb{Z}
$$

inducing a natural transformation of homology functors $t h_{*}: M S O_{*}(-) \rightarrow H_{*}(-; \mathbb{Z})$, which can be described geometrically as follows. Any element of $M S O_{k}(X)$ is represented by a closed oriented $k$-manifold $M$ and a map $f: M \rightarrow X$.

Lemma 3.2.1. The homomorphism $t h_{*}$ sends the class $\left[\left(M^{k} \rightarrow X\right)\right]$ to the homology class given by the image of the fundamental class of $M$; i.e., $t h_{*}([(M \stackrel{f}{\rightarrow} X)])=f_{*}[M] \in H_{k}(X ; \mathbb{Z})$.

Proof. Let $\mu_{M}$ denote the fundamental cohomology class of $M$. Let $u$ denote the Thom class of $-T M$. It suffices to show that $\left\langle t h_{*}[M \stackrel{i d}{\rightarrow} M], \mu_{M}\right\rangle=1$. Moreover, by the naturality of Thom classes and the definition of the Kronecker pairing, it suffices to show that the composition

$$
\Sigma^{\infty} p t_{+} \stackrel{P T_{(M \rightarrow p t)}}{\longrightarrow} \mathbf{T h}(-T M) \stackrel{\Delta}{\longrightarrow} \mathbf{T h}(-T M) \wedge M_{+} \stackrel{u \wedge \mu_{M}}{\longrightarrow} \mathbf{H} \mathbb{Z} \wedge \mathbf{H} \mathbb{Z} \stackrel{\text { prod }}{\longrightarrow} \mathbf{H} \mathbb{Z}
$$

represents $1 \in H^{0}(p t ; \mathbb{Z})$. This holds because $\Delta^{*}\left(u \wedge \mu_{M}\right)=u \mu_{M}$ and $P T_{(M \rightarrow p t)}^{*}(u \cdot-)$ is integration over $M$.

Lemma 3.2.2. Let $c \in \operatorname{Sym}\left(H^{>0}(\operatorname{MTSO}(d) ; \mathbb{Z})\right) \subset H^{*}\left(\Omega^{\infty} \operatorname{MTSO}(d) ; \mathbb{Z}\right)$ be a polynomial in the universal generalised MMM classes. Then, $c^{\sharp}\left(E^{n+d} \stackrel{\pi}{\rightarrow} B^{n}\right)=\left\langle c, t h_{*}\left[\alpha_{\pi}\right]\right\rangle$.

Proof. We have

$$
\begin{aligned}
c^{\sharp}(E \rightarrow B) & =\left\langle\left(\alpha_{\pi}\right)^{*} c,[B]\right\rangle \\
& =\left\langle c,\left(\alpha_{\pi}\right)_{*}[B]\right\rangle \\
& =\left\langle c, t h_{*}\left[\alpha_{\pi}\right]\right\rangle,
\end{aligned}
$$

where the first equality is immediate from Proposition 3.1 .2 , and the last equality comes from Lemma3.2.1 


\section{The IMAGE of $\alpha$ AND THE PROOF OF THEOREMC}

We now show how Theorem B together with Ebert's results on the algebraic independence of the generalised MMM classes implies Theorem $\mathbb{C}$ and the $B u n_{d}^{\mathbb{C}}$ part of Theorem $\mathrm{E}$

4.1. The real oriented case. In studying the image of $\alpha$ it will be convenient to dualize and instead study the kernel of the linear map

$$
\alpha^{\vee}: \operatorname{Hom}\left(\text { Bun }_{d}, \mathbb{Q}\right) \rightarrow \operatorname{Hom}\left(\operatorname{MSO}_{*}\left(\Omega^{\infty} \mathbf{M T S O}(d)\right), \mathbb{Q}\right) .
$$

According to Proposition 3.1.1 the space of generalised MMM classes $\mathscr{C}_{d} \otimes \mathbb{Q}$ can be canonically identified with a subspace of $\operatorname{Hom}\left(M_{S} O_{*}\left(\Omega^{\infty} \mathbf{M T S O}(d)\right), \mathbb{Q}\right)$. By Theorem $B$ an element $x$ in the subspace $\mathscr{C}_{d} \otimes \mathbb{Q}$ lies in the image of

$$
(\widetilde{T o t})^{\vee}: \operatorname{Hom}\left(M S O_{*+d}(p t), \mathbb{Q}\right) \rightarrow \operatorname{Hom}\left(M_{S O}\left(\Omega^{\infty} \operatorname{MTSO}(d)\right), \mathbb{Q}\right) .
$$

if and only if $x$ is of the form $\widehat{X}$ for $X$ in the space $N P_{d}$ of order $d$ near-primitives (restricted from $B S O$ to $B S O(d)$ ).

Lemma 4.1.1. The restriction of $\alpha^{\vee}$ to the subspace $\mathscr{C}_{d} \otimes \mathbb{Q}$ is injective when $d$ is even; when $d$ is odd there is a kernel $K$ and it is the ideal in $\mathscr{C}_{d} \otimes \mathbb{Q}$ generated by the generalised MMM classes of the form $\widehat{X}$ for $X$ a homogeneous component of the Hirzebruch L-class.

Proof. First suppose $d$ is even. Ebert's algebraic independence result [Ebe11a, Theorem B] asserts that for any nonzero class $x \in \mathscr{C}_{d} \otimes \mathbb{Q}$ (which we take to be of degree $m$ ) there exists a $d$-manifold $Z$ such that the evaluation of $x$ on the tautological $Z$-bundle over $B \operatorname{Diff}(Z)$ is nontrivial in rational cohomology. Since the map $M S O_{*}(B \operatorname{Diff}(Z)) \otimes \mathbb{Q} \rightarrow H_{*}(Z ; \mathbb{Q})$ is surjective, there exist a closed oriented $m$-manifold $M$ and a map $f: M \rightarrow B \operatorname{Diff}(Z)$ such that $\left\langle f^{*} x,[M]\right\rangle$ is nonzero. Hence $x^{\sharp}$ evaluates nontrivially on the induced $Z$-bundle over $M$ and so $\alpha^{*}\left(x^{\sharp}\right) \neq 0$.

When $d$ is odd, Ebert's theorem asserts the existence of a manifold $Z$ for which $x$ is nonzero on $B \operatorname{Diff}(Z)$ as above if and only if if $x$ is nonzero in the quotient $\mathscr{C}_{d} / K$, where $K$ is the ideal generated by the classes of the form $\widehat{X}$ for $X$ a homogeneous component of the Hirzebruch $L$-class.

Theorem Cnow follows immediately from the above lemma together with Theorem $B$, which we shall prove below in Section 5 .

4.2. The complex case. The argument in this case is identical to the argument in the real case. The injectivity of $\left.\left(\alpha^{\mathbb{C}}\right)^{\vee}\right|_{\mathscr{C}_{d}^{\mathbb{C}} \otimes \mathbb{Q}}$ follows from [Ebe11a, Theorem C]. In this setting there is no distinction between even and odd $d$.

4.3. The Madsen-Weiss Theorem and $d=2$. The discussion here is not necessary for any of the results in this paper, but we include it for completeness. When $d=2$ the Madsen-Weiss theorem [MW07] implies that the image of $\alpha$ is quite large. Let $\pi_{0}$ denote the group of path components of $\Omega^{\infty} \mathbf{M T S O}(2)$. (This group is isomorphic to $\mathbb{Z}$ and corresponds to the genus of the fibres of a surface bundle.)

Proposition 4.3.1. The map Bun $2 \stackrel{\alpha}{\rightarrow} \operatorname{MSO}_{*}(\Omega \operatorname{MTSO}(2)) \rightarrow \operatorname{MSO}_{*}\left(\Omega^{\infty} \operatorname{MTSO}(2)\right)_{\pi_{0}}$ is surjective. 
Proof. Let $\Sigma_{g, 1}$ be a compact connected oriented surface of genus $g$ with one boundary component, and let Diff ${ }_{\partial}\left(\Sigma_{g, 1}\right)$ denote the topological group of orientation-preserving diffeomorphisms that restrict to the identity on the boundary. Gluing the boundary of the surface to one boundary a torus with two boundary components gives a stabilisation map $\operatorname{Diff}_{\partial}\left(\Sigma_{g, 1}\right) \rightarrow \operatorname{Diff}_{\partial}\left(\Sigma_{g+1,1}\right)$.

For each $g$ there is a universal bundle $\pi(g): E_{g} \rightarrow B \operatorname{Diff}_{\partial}\left(\Sigma_{g, 1}\right)$ and thus an associated map

$$
\alpha_{\pi(g)}: B \operatorname{Diff}_{\partial}\left(\Sigma_{g, 1}\right) \rightarrow \Omega^{\infty} \mathbf{M T S O}(2)
$$

One can adjust these maps to all land in the identity component $\Omega_{0}^{\infty} \mathbf{M T S O}(2)$ by translating and then check that they are compatible with the above stabilisation up to homotopy. By the Madsen-Weiss Theorem [MW07] (see also [GTMW09] and [GRW10a]), the resulting map

$$
\alpha_{\infty}: \operatorname{hocolim}_{g \rightarrow \infty} B \operatorname{Diff}_{\partial}\left(\Sigma_{g, 1}\right) \rightarrow \Omega_{0}^{\infty} \mathbf{M T S O}(2)
$$

is an isomorphism in any generalised cohomology theory, including $\mathrm{MSO}_{*}(-)$.

Since $B$ Diff ${ }_{\partial}\left(\Sigma_{g, 1}\right)$ classifies bundles with fibre $\Sigma_{g, 1}$ (and trivial boundary bundle), the map $\alpha: \mathrm{Bun}_{2} \rightarrow \mathrm{MSO}_{*}\left(\Omega^{\infty} \mathbf{M T S O}(2)\right)$, when restricted to the subspace $B u n_{2}^{\prime} \subset B u n_{2}$ of bundles that admit a framed section, factors as

$$
\operatorname{Bun}_{2}^{\prime} \rightarrow \operatorname{MSO}_{*}\left(\coprod_{g} B \operatorname{Diff}\left(\Sigma_{g, 1}\right)\right) \rightarrow \operatorname{MSO}_{*}\left(\Omega^{\infty} \mathbf{M T S O}(2)\right) .
$$

It now follows from the Madsen-Weiss Theorem that $\alpha$ is surjective onto the $\pi_{0}$-coinvariants.

When $d$ is even and greater than 4, Galatius and Randal-Williams have announced in [GRW10b] a result along the lines of the Madsen-Weiss Theorem. This result implies a large lower bound on the size of the image of $\alpha$.

\section{REFORMULATION IN TERMS OF STABLE HOMOTOPY}

Here we give the proofs of Theorem $B$ and Theorem $E$ part (1) by reformulating the factorisation question at the level of spectra and carrying out a rational calculation.

5.1. The real case. The problem is to decide which generalised MMM classes $x \in \mathscr{C}_{d} \otimes \mathbb{Q}$ have the property that the associated characteristic number $x^{\sharp}$ admits a factorisation

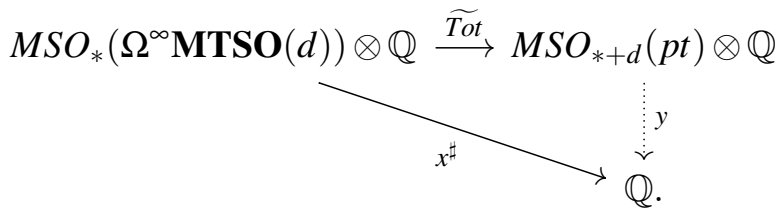

We now reformulate this diagram at the level of spectra. The set of linear maps $M S O_{*}(p t) \otimes \mathbb{Q} \rightarrow \mathbb{Q}$ is in canonical bijection with $H^{*}(\mathbf{M S O} ; \mathbb{Q})$ and thus with homotopy classes of maps MSO $\rightarrow \mathbf{H} \mathbb{Q}$. Given a bundle $\pi: E^{n+d} \rightarrow B^{n}$, the class $\left[\alpha_{\pi}\right] \in \operatorname{MSO}_{n}\left(\Omega^{\infty} \mathbf{M T S O}(d)\right)$ is represented by a degree $n$ map of spectra

$$
\mathbf{S}^{0} \rightarrow \mathbf{M S O} \wedge \Omega^{\infty} \mathbf{M T S O}(d)_{+} .
$$

As described earlier, the oriented bordism class of the total space of the bundle is represented by the homotopy class of the composition

$$
\mathbf{S}^{0} \stackrel{\alpha_{\pi}}{\rightarrow} \mathbf{M S O} \wedge \Omega^{\infty} \mathbf{M T S O}(d)_{+} \stackrel{i d \wedge \sigma}{\rightarrow} \mathbf{M S O} \wedge \mathbf{M T S O}(d) \rightarrow \mathbf{M S O} \wedge \text { MSO } \stackrel{\text { prod }}{\rightarrow} \text { MSO }
$$

which is a map of degree $n+d$. It is elementary to see that, given a class $x \in H^{n}\left(\Omega^{\infty} \operatorname{MTSO}(d) ; \mathbb{Q}\right)$, the characteristic number $x^{\sharp}\left(E^{n+d} \stackrel{\pi}{\rightarrow} B^{n}\right)$ is represented by the homotopy class of the composition

$$
\mathbf{S}^{0} \stackrel{\left[\alpha_{\pi}\right]}{\rightarrow} \mathbf{M S O} \wedge \Omega^{\infty} \mathbf{M T S O}(d)_{+} \stackrel{\text { th }}{\rightarrow} \mathbf{H} \mathbb{Q} \wedge \Omega^{\infty} \mathbf{M T S O}(d)_{+} \stackrel{1 \wedge x}{\rightarrow} \mathbf{H} \mathbb{Q} \wedge \mathbf{H} \mathbb{Q} \stackrel{\text { prod }}{\rightarrow} \mathbf{H} \mathbb{Q}
$$


which is of degree zero and hence gives an element of $\mathbb{Q}$. We therefore have the following.

Lemma 5.1.1. The problem of factorisation in diagram (5.1.1) is thus equivalent to the problem of deciding for which $x$ there exists some $y: \mathbf{M S O} \rightarrow \mathbf{H} \mathbb{Q}$ such that the diagram

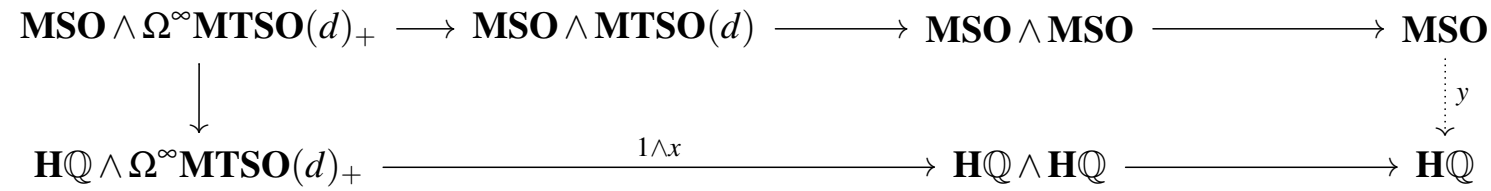

commutes up to homotopy.

Proof of Theorem $B$ The counter-clockwise composition in the diagram of Lemma 5.1.1 represents a class in

$$
H^{*}\left(\mathbf{M S O} \wedge \Omega^{\infty} \mathbf{M T S O}(d) ; \mathbb{Q}\right) \cong H^{*}(\operatorname{MSO} ; \mathbb{Q}) \otimes H^{*}\left(\Omega^{\infty} \operatorname{MTSO}(d) ; \mathbb{Q}\right)
$$

that one immediately identifies as $t h \otimes x$. Thus existence of such a $y$ is equivalent to existence of a class $y \in H^{*}(\mathbf{M S O} ; \mathbb{Q})$ that pulls back along the top row to $t h \otimes x$. By Lemma 6.3.1 (proved in the sequel), this is equivalent to $y$ being the image under the Thom isomorphism of a class $y^{\prime} \in H^{*}(B S O ; \mathbb{Q})$ that is near-primitive of order $d$ and whose restriction to $B S O(d)$ maps to $x$ under the Thom isomorphism. This concludes the proof of Theorem B

5.2. The complex case. Suppose now that $\pi: E^{n+d} \rightarrow B^{n}$ is a holomorphic fibre bundle in the category of closed almost complex manifolds (the superscripts refer to the complex dimensions). Then the fibrewise tangent bundle has structure group $U(d)$ and we have a classifying map

$$
\alpha_{\pi}: B \rightarrow \Omega^{\infty} \mathbf{M T U}(d) .
$$

The bordism class of this map represents an element $\left[\alpha_{\pi}\right] \in M U_{2 n}\left(\Omega^{\infty} \mathbf{M T U}(d)\right)$, and this defines an additive map $\alpha^{\mathbb{C}}: B u n_{d}^{\mathbb{C}} \rightarrow M U_{*}\left(\Omega^{\infty} \mathbf{M T U}(d)\right)$. As in the oriented case, there is a map

$$
\widetilde{\text { Tot }}: \operatorname{MU}_{*}\left(\Omega^{\infty} \mathbf{M T U}(d)\right) \rightarrow M U_{*+2 d}(p t)
$$

The total space $E$ represents an element of $M U_{n+2 d}(p t)$ and $\widetilde{\text { Tot }}:\left[\alpha_{\pi}\right] \mapsto[E]$.

We now consider the diagram:

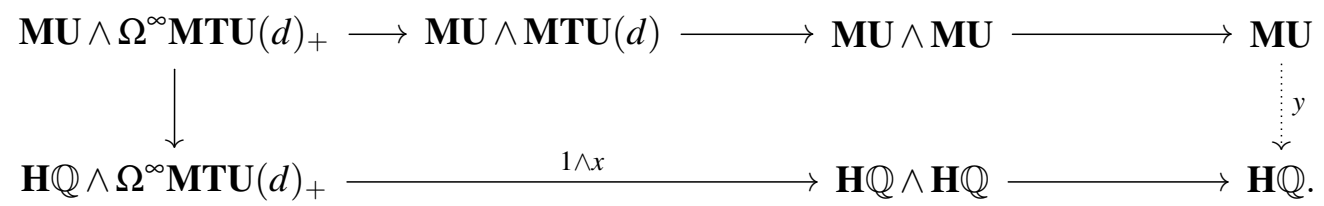

Proof of Theorem E part (1). As in the real case, existence of a $y$ making this diagram commute up to homotopy is equivalent to existence of a class $y \in H^{*}(\mathbf{M U} ; \mathbb{Q})$ that pulls back along the top row to $t h \otimes x$, and by Lemma 6.3.1 (proved in the sequel), this is equivalent to $y$ being the image under the Thom isomorphism of a class $y^{\prime} \in H^{*}(B U ; \mathbb{Q})$ that is near-primitive of order $2 d$ and whose restriction to $B U(d)$ maps to $x$ under the Thom isomorphism. This concludes the proof of the part of Theorem Ereferring to maps out of $M U_{*}\left(\Omega^{\infty} \mathbf{M T U}(d)\right)$.

\section{NEAR-PRIMITIVES}

In this section we shall prove Theorem $\mathrm{A}$ which gives a complete description of the nearprimitives in $H^{*}(B S O ; \mathbb{Q})$ and $H^{*}(B U ; \mathbb{Q})$. We also prove Lemma 6.3.1 showing that near-primitives of order $d$ on $B S O$ or order $2 d$ on $B U$ can be effectively detected by restricting to $B S O(d)$ or $B U(d)$ respectively. 
6.1. Recollections. Recall that, as an algebra, $H^{*}(B U ; \mathbb{Q})$ is a polynomial algebra with a single generator in each even degree. These generators can be taken to be the Chern classes $c_{i} \in H^{2 i}(B U ; \mathbb{Q})$. In terms of these generators, the coproduct is determined by the formula

$$
\Delta\left(c_{i}\right)=\sum_{i=0}^{n} c_{i} \otimes c_{n-i}
$$

(where $c_{0}=1$ ). The cohomology of $B U(d)$ is isomorphic to $\mathbb{Q}\left[c_{1}, \ldots, c_{d}\right]$, and the restriction from $B U$ to $B U(d)$ kills the ideal generated by $\left\{c_{d+1}, c_{d+2}, \ldots\right\}$.

In the real case, $H^{*}(B S O ; \mathbb{Q})$ is a polynomial algebra with generators the Pontrjagin classes $p_{i} \in H^{4 i}(B S O ; \mathbb{Q})$. The coproduct is determined by the formula

$$
\Delta\left(p_{i}\right)=\sum_{i=0}^{n} p_{i} \otimes p_{n-i}
$$

(where $p_{0}=1$ ). The cohomology of $B S O(d)$ is isomorphic to $\mathbb{Q}\left[p_{1}, \ldots, p_{\lfloor d / 2\rfloor}\right]$ if $d$ is odd and $\mathbb{Q}\left[p_{1}, \ldots, p_{d / 2}, e\right] /\left(e^{2}=p_{d / 2}\right)$ if $d$ is even. The restriction from $B S O$ to $B S O(d)$ kills the ideal generated by $\left\{p_{\lfloor d / 2\rfloor+i}\right\}_{i \geq 1}$.

Let $H$ be either $H^{*}(B U ; \mathbb{Q})$ or $H^{*}(B S O ; \mathbb{Q})$. To describe near-primitives in $H$ it is convenient to work with a set of primitive generators. By the structure theorem for Hopf algebras, the dual of $H$ is polynomial, from which it follows that the space of primitives in degree $i$ has dimension 1 if $H^{i}$ is non-zero and has dimension zero otherwise. Thus there is a primitive $Q_{i}$ (unique up to scalars) in each degree $2 i$ in the complex case, and $4 i$ in the real case. These primitives can be taken to be the components of the Chern character and Pontrjagin character respectively. The primitives freely generate $H$ as a graded commutative algebra, so that $H$ is the polynomial algebra in $Q_{1}, Q_{2}, \ldots$.

Since the coproduct $\Delta$ is connected, if $x$ is an element of positive degree then $\Delta(x)$ is equal to $x \otimes 1+1 \otimes x$ plus terms of bidegree $(p, q)$ with $p, q>0$. For convenience, we shall work with the reduced coproduct $\bar{\Delta}$ defined by $\bar{\Delta}=\Delta-1 \otimes i d-i d \otimes 1$ in positive degrees, and $\bar{\Delta}=0$ on elements of degree zero. Observe that if $x \in H$ is an element of degree $\geq d$ then $x$ is a near-primitive of order $d$ if and only it is in the kernel of the map

$$
H \stackrel{\bar{\Delta}}{\rightarrow} H \otimes H \stackrel{i d \otimes \text { proj }}{\longrightarrow} H \otimes H^{\geq d},
$$

where proj projects the summands in degree $<d$ to zero.

6.2. Classification of near-primitives in $H^{*}(B S O ; \mathbb{Q})$ and $H^{*}(B U ; \mathbb{Q})$. We now prove Theorem A. restated below. We continue to let $H$ denote either $H^{*}(B S O ; \mathbb{Q})$ or $H^{*}(B U ; \mathbb{Q})$.

Theorem 6.2.1. The space of near-primitives of order $d$ in $H$ has a basis consisting of those monomials in the primitives $Q_{i}$ such that all proper factors are of degree strictly less than $d$. Explicitly, the degree $m$ component of the space is equal to the degree $m$ part of $\mathbb{Q}\left[Q_{i} \mid m-d<\right.$ $\left.\left|Q_{i}\right|<d\right] \oplus \mathbb{Q} Q_{m}$.

In a fixed degree $m$ (which we take to be even since $H$ is concentrated in even degrees), let us consider how the space of near-primitives depends on $d$. If $d<m / 2$ then the only near-primitives are the ordinary primitives since the set of $i$ for which $m-d<\left|Q_{i}\right|<d$ is empty. When $d=m / 2$, if there is a $Q_{i}$ of degree $d-1$ then $Q_{i}^{2}$ is a near-primitive that is not primitive. As $d$ increases more near-primitives appear, until $d=m$ at which point everything is in the kernel of the map (6.1.1). In general, the dimension of the space of near-primitives in degree $m$ can easily be recovered from the Poincaré series for the polynomial algebra on $\left\{Q_{i}: m-d<\left|Q_{i}\right|<d\right\}$.

Theorem 6.2.1 follows directly from the following property of the coproduct in $H$. 
Lemma 6.2.2. Let $x \in \mathbb{Q}\left[Q_{1}, Q_{2}, \ldots\right]$. The monomial summands of $\bar{\Delta}(x)$ correspond to proper factorizations of summands of $x$. In particular, $\bar{\Delta}(x)$ has a summand $y \otimes z$ with $|z|=k$ if and only if $x$ has a summand with a degree $k$ factor.

Proof. Since each $Q_{i}$ is primitive, we can easily calculate the coproduct of a monomial explicitly:

$$
\Delta\left(Q_{1}^{f_{1}} Q_{2}^{f_{2}} \cdots\right)=\sum_{i_{1}, i_{2}, \ldots}\left(\left(\begin{array}{c}
f_{1} \\
i_{1}
\end{array}\right)\left(\begin{array}{c}
f_{2} \\
i_{2}
\end{array}\right) \cdots\right)\left(Q_{1}^{i_{1}} Q_{2}^{i_{2}} \cdots\right) \otimes\left(Q_{1}^{f_{1}-i_{1}} Q_{2}^{f_{2}-i_{2}} \cdots\right),
$$

where the summation runs over all sequences $i_{1}, i_{2}, \ldots$ with $0 \leq i_{1} \leq f_{1}, 0 \leq i_{2} \leq f_{2}, \ldots$. Hence if the monomial $\left(Q_{1}^{f_{1}} Q_{2}^{f_{2}} \cdots\right)$ has a degree $k$ factor, say $Q_{1}^{i_{1}} Q_{2}^{i_{2}} \cdots$, then $\Delta\left(Q_{1}^{f_{1}} Q_{2}^{f_{2}} \cdots\right)$ will have a summand $\left(Q_{1}^{i_{1}} Q_{2}^{i_{2}} \cdots\right) \otimes\left(Q_{1}^{f_{1}-i_{1}} Q_{2}^{f_{2}-i_{2}} \cdots\right)$ (with coefficient $\left.\left(\begin{array}{c}f_{1} \\ i_{1}\end{array}\right)\left(\begin{array}{l}f_{2} \\ i_{2}\end{array}\right) \cdots\right)$. Consequently, $\bar{\Delta}\left(Q_{1}^{f_{1}} Q_{2}^{f_{2}} \cdots\right)$ will have a summand $y \otimes z$ with $|z|=k$.

Conversely, if $\bar{\Delta}\left(Q_{1}^{f_{1}} Q_{2}^{f_{2}} \cdots\right)$ has a summand $y \otimes z$ with $|z|=k$, then $z$ must be $\left(Q_{1}^{i_{1}} Q_{2}^{i_{2}} \cdots\right)$ for some sequence $i_{1}, i_{2}, \ldots$ with $i_{1} \leq f_{1}, i_{2} \leq f_{2}$, etc. Hence $\left(Q_{1}^{i_{1}} Q_{2}^{i_{2}} \cdots\right)$ is a factor of $\left(Q_{1}^{f_{1}} Q_{2}^{f_{2}} \cdots\right)$.

From the above formula it is also clear that two different monomials $Q_{1}^{f_{1}} Q_{2}^{f_{2}} \cdots$ and $Q_{1}^{g_{1}} Q_{1}^{g_{2}} \ldots$ cannot have the same summand in their images under $\Delta$, since adding the exponents reveals the source of the summand.

6.3. Restricting to $B U(d)$ or $B S O(d)$. Let $i$ denote either of the inclusions $B S O(d) \hookrightarrow B S O$ or $B U(d) \hookrightarrow B U$. Let proj denote either the projection $H^{*}(B S O ; \mathbb{Q}) \rightarrow H^{\geq d}(B S O ; \mathbb{Q})$ or the projection $H^{*}(B U ; \mathbb{Q}) \rightarrow H^{\geq 2 d}(B U ; \mathbb{Q})$. The following lemma asserts that near-primitives of order $d$ on $B S O$ can be faithfully detected on $B S O(d)$ in an appropriate sense, and likewise for near-primitives of order $2 d$ and $B U(d)$.

Lemma 6.3.1. There is an equality of kernels,

$$
\operatorname{ker}\left(1 \otimes\left(i^{*} \circ \operatorname{proj}\right)\right) \circ \bar{\Delta}=\operatorname{ker}(1 \otimes \operatorname{proj}) \circ \bar{\Delta}
$$

Proof. We first prove the complex case and then describe the modifications needed for the real case. Let $x \in \operatorname{ker}\left(1 \otimes\left(i^{*} \circ \operatorname{proj}\right)\right) \circ \bar{\Delta}$, i.e., $x \in \mathbb{Q}\left[Q_{1}, Q_{2}, \ldots\right]$ is such that the only summands $y \otimes z$ in $\bar{\Delta}(x)$ in which $|z| \geq 2 d$ have $z \in \operatorname{ker}\left(i^{*}\right)=\left\langle c_{d+1}, c_{d+2}, \ldots\right\rangle$. If $x$, as a polynomial in $Q_{1}, Q_{2}, \ldots$, has a monomial summand that features $Q_{j}$ as a factor with $\left|Q_{j}\right| \geq 2 d$, then, by Lemma 6.2.2, that summand will give rise to a term in $\bar{\Delta}(x)$ of the form $w \otimes Q_{j}$. Since $\left|Q_{j}\right| \geq 2 d$ we would need $Q_{j} \in\left\langle c_{d+1}, c_{d+2}, \ldots\right\rangle$ to avoid contradicting the hypotheses on $x$. However, writing the primitives as polynomials in the Chern classes, $Q_{j}$ contains $c_{1}^{j}$ as a summand with coefficient $1 /(j !)$ by Newton's identities, so $Q_{j} \notin\left\langle c_{d+1}, c_{d+2}, \ldots\right\rangle$. Hence $x$ can have no summand admitting a proper factor $Q_{j}$ with $\left|Q_{j}\right| \geq 2 d$. Hence $\bar{\Delta}(x)$ features no summands $y \otimes z$ with $z \in\left\langle c_{d+1}, c_{d+2}, \ldots\right\rangle$, and so $x \in \operatorname{ker}(1 \otimes \operatorname{proj}) \circ \bar{\Delta}$. The opposite inclusion is trivial.

The proof in the real case is nearly identical. The only difference is that now $Q_{j}$ sits in degree $4 j$ and, when written as a polynomial in the Pontrjagin classes, contains a term $p_{j}^{1}$ with coefficient $1 /(j !)$.

\section{REFERENCES}

[CFT] Thomas Church, Benson Farb, and Matthew Thibault, On the geometric nature of characteristic classes of surface bundles, arXiv:1103.0218.

[Ebe11a] Johannes Ebert, Algebraic independence of generalized MMM-classes, Algebr. Geom. Topol. 11 (2011), no. 1, 69-105.

[Ebe11b] _ A vanishing theorem for characteristic classes of odd-dimensional manifold bundles, To appear in Journal fur die Reine und Angewandt Mathematik, 2011. 
[GRW10a] Søren Galatius and Oscar Randal-Williams, Monoids of moduli spaces of manifolds, Geom. Topol. 14 (2010), no. 3, 1243-1302.

[GRW10b] _ Monoids of moduli spaces of manifolds, II, Oberwolfach Reports 42 (2010), 18-21.

[GTMW09] Søren Galatius, Ulrike Tillmann, Ib Madsen, and Michael Weiss, The homotopy type of the cobordism category, Acta Math. 202 (2009), no. 2, 195-239.

[Mil86] Edward Y. Miller, The homology of the mapping class group, J. Differential Geom. 24 (1986), no. 1, 1-14.

[MM65] John W. Milnor and John C. Moore, On the structure of hopf algebras, The Annals of Mathematics 81 (1965), no. 2, pp. 211-264.

[Mor87] Shigeyuki Morita, Characteristic classes of surface bundles, Invent. Math. 90 (1987), no. 3, 551-577.

[Mum83] David Mumford, Towards an enumerative geometry of the moduli space of curves, Arithmetic and geometry, Vol. II, Progr. Math., vol. 36, Birkhäuser Boston, Boston, MA, 1983, pp. 271-328.

[MW07] Ib Madsen and Michael Weiss, The stable moduli space of Riemann surfaces: Mumford's conjecture, Ann. of Math. (2) 165 (2007), no. 3, 843-941. 